\title{
A Study of the Antigenic Relationships of Isolates of Trypanosoma brucei Collected from a Herd of Cattle Kept in One Locality for Five Years
}

\author{
By A. R. GRAY \\ The Nigerian Institute for Trypanosomiasis Research, \\ Vom, Benue-Plateau State, Nigeria \\ (Accepted for publication 5 May 1970)
}

SUMMAR Y

During surveys of the prevalence of trypanosomiasis, 37 isolates of trypanosomes of the subgenus Trypanozoon were collected over a period of five years from a herd of cattle kept on a farm in an area in Nigeria where human and animal trypanosomiasis is endemic. Thirty-three were typical isolates of Trypanosoma brucei but four were unusual in several respects and their identity was uncertain.

The antigenic relationships of the trypanosomes were determined by agglutination tests using antisera to the predominant variant antigens of each isolate. Comparisons of groups of isolates obtained in two of the surveys showed that some of the animals in the herd were infected with antigenically related trypanosomes. Four isolates obtained in one survey had antigens in common and probably belonged to one trypanosome strain, while 26 isolates obtained in another survey seemed to have been derived from three different strains. At least ten animals examined in the latter survey were infected with two strains of Trypanosoma brucei at the same time. Comparisons of trypanosomes obtained in successive surveys showed that one isolate was very closely related to four isolates obtained from different animals two years later. Agglutination and neutralization tests, and a study of variant antigen production by clones prepared from the first isolate and one of the later group of four isolates, established the antigenic similarity of these trypanosomes and provided direct evidence of the long-term antigenic stability of a trypanosome strain under field conditions. Apart from this notable exception, there was little antigenic relationship among the trypanosomes isolated from year to year and at least seven antigenically distinct strains of $T$. brucei were isolated from the herd in the five-year observation period.

\section{INTRODUCTION}

The development of improved methods for determining the antigenic relationships of isolates of Trypanosoma brucei and T. rhodesiense (Cunningham \& Vickerman, I962; Gray, I966) and for the long-term preservation of trypanosomes at low temperatures (Walker, 1966) has facilitated immunological studies on several aspects of the epidemiology of African trypanosomiasis. Although there are major antigenic differences between strains of T. brucei isolated in widely separated areas, close relationships frequently occur among organisms isolated in restricted geographical localities (Broom \& Brown, 1940; Cunningham \& Vickerman, 1962; Gray, 1966). Comparisons of the variant antigens of strains of $T$. brucei and $T$. rhodesiense have indicated that it 
may sometimes be possible to differentiate between these morphologically identical species by serological tests (Weitz, I962; Taylor, 1968).

Such findings suggest that it might eventually be possible to immunize animals against trypanosomiasis in certain areas and to use serological methods to determine whether pleomorphic trypanosomes of the subgenus Trypanozoon (Hoare, 1964) isolated from animals in the course of epidemiological studies are Trypanosoma brucei, $T$. rhodesiense or, possibly, T. gambiense. At present, $T$. brucei can be distinguished from the other two species with certainty only by its inability to infect man, while $T$. rhodesiense and $T$. gambiense are identified on the basis of their geographical origin, sensitivity to certain trypanocidal drugs and pathogenicity in man and experimental animals (Ashcroft, 1959). It is generally acknowledged that much more information on the numbers of antigenically different strains of these three species occurring in selected areas and on the antigenic stability of trypanosome strains in the field is needed before such suggestions can be properly evaluated (Lumsden, 1965).

In I96I an exceptionally high prevalence of trypanosomiasis was discovered in a herd of cattle kept on a government farm in Benue Province, Nigeria (Godfrey, Leach \& Killick-Kendrick, 1964). Variations in the prevalence of trypanosome infections in this herd were studied on four further occasions during the next five years and numerous trypanosome isolates, including 37 classified in the subgenus Trypanozoon, were collected for study in the laboratory. This paper describes an investigation to find the number of antigenically different strains of this subgenus which infected the cattle in the five-year observation period and a search for evidence of antigenic stability in trypanosome strains in their natural environment.

\section{METHODS}

Origin and ecology of the trypanosomes. The trypanosomes were isolated from a herd of cattle kept on a government farm at Raav, in Benue Province, Nigeria. They were pleomorphic organisms belonging to the subgenus Trypanozoon and were identified on the basis of their morphology and bovine origin as Trypanosoma brucei.

The farm consisted of 6000 acres of land south of the River Benue in the Guinea Savannah Vegetation Zone(Keay, I953). Low numbers of the tsetse flies Glossina palpalis and $G$. tachinoides occurred in the area and human and animal trypanosomiasis was endemic. No attempt was made to prevent trypanosome infections in the cattle, but when necessary, severely affected animals were treated with therapeutic trypanocidal drugs such as homidium chloride, B.Vet.C., and diminazene aceturate, B.Vet.C.

The trypanosomes were collected during surveys of the prevalence of trypanosomiasis in the animals, carried out between November 196I and April 1966 by methods described by Godfrey \& Killick-Kendrick (1961). Five surveys were made and many animals were found to be infected with Trypanosoma vivax, T. congolense and T. brucei. A full account of variations in the prevalence of trypanosome infections found in the first three surveys has been given elsewhere (Godfrey et al. I964). A summary of the findings in all five surveys in relation to T. brucei is shown in Table I.

Isolation and adaptation of the trypanosomes. The trypanosomes were isolated by inoculating blood from infected cattle into white rats. Each isolate was identified by the number of the survey in which it was collected and by the number of the rat inoculated with blood from the bovine host. Thus, isolate 5/28 was collected in survey 5 in the 28 th rat inoculated with ox blood. Only two of the 20 isolates of Trypanosoma 
brucei found in the herd in survey 5 and none of the three isolates infecting the animals in survey 9 were available for study, but most of the organisms found in the later surveys 17,22 and 30 were examined serologically (Table I).

Thirty-two of the isolates were collected from animals with pure Trypanosoma brucei infections, but three were obtained from cattle infected with $T$. brucei and $T$. vivax and two from animals infected with $T$. bruce $i$ and $T$. congolense. Pure lines of $T$. brucei were obtained from mixed isolates of $T$. brucei and $T$. vivax by passaging the trypanosomes repeatedly in rats, which are not readily infected with $T$. vivax, and from mixtures of $T$. brucei and $T$. congolense by preparing clones of the former species by the method of Inoki (1960).

Table I. Trypanosoma brucei infections found in five surveys of trypanosomiasis in a herd of cattle and the number of isolates of the trypanosome examined serologically

\begin{tabular}{|c|c|c|c|c|c|c|c|c|}
\hline \multirow[b]{2}{*}{$\begin{array}{c}\text { No. } \\
\text { of } \\
\text { survey }\end{array}$} & \multirow[b]{2}{*}{$\begin{array}{l}\text { Date } \\
\text { of } \\
\text { survey }\end{array}$} & \multirow[b]{2}{*}{$\begin{array}{c}\text { No. of } \\
\text { animals } \\
\text { examined }\end{array}$} & \multicolumn{4}{|c|}{ Types of infections } & \multirow[b]{2}{*}{$\begin{array}{l}\text { No. of } \\
\text { animals } \\
\text { infected } \\
\text { with } \\
\text { T. brucei }\end{array}$} & \multirow[b]{2}{*}{$\begin{array}{l}\text { No. of } \\
\text { isolates } \\
\text { examined } \\
\text { sero- } \\
\text { logically }\end{array}$} \\
\hline & & & $\begin{array}{l}\text { T. brucei } \\
\text { alone }\end{array}$ & $\begin{array}{c}\text { T. brucei } \\
\text { and } \\
T . \text { vivax }\end{array}$ & $\begin{array}{c}T . \text { brucei } \\
\text { and } T \text {. } \\
\text { congolense }\end{array}$ & $\begin{array}{c}\text { T. brucei, } \\
T . \text { vivax } \\
\text { and } T . \\
\text { congolense }\end{array}$ & & \\
\hline 5 & 23. xi. $6 I$ & 99 & 0 & 4 & I3 & 3 & 20 & 2 \\
\hline 9 & 8. xi. 62 & 77 & $\mathbf{I}$ & I & I & 0 & 3 & 0 \\
\hline 17 & 28. xi. 63 & 95 & 4 & 0 & 2 & 0 & 6 & 6 \\
\hline 22 & 27. i. 65 & 100 & I & 0 & 0 & 0 & I & I \\
\hline 30 & 26. iv. 66 & 107 & 29 & I & I & 0 & $3 I$ & 28 \\
\hline
\end{tabular}

A few of the trypanosomes were very pathogenic when first isolated from the cattle and gave rise to acute infections and massive parasitaemias in rats and mice within 3 to 4 days, but in most cases the primary rodent infections were mild. As heavy suspensions of trypanosomes were required for serological tests, the less virulent isolates were passaged repeatedly at short intervals in rats and mice until they adapted to these hosts. They were then frozen and stored at $-80^{\circ}$ as antigens for agglutination tests (Cunningham \& Vickerman, 1962). In three instances, the degree of adaptation induced in isolates by prolonged passage was very low and the trypanosomes were frozen and stored in an incompletely adapted state. Fuller details of the number of times particular isolates were passaged in rats and mice are given below where relevant.

\section{Determination of the antigenic relationships of the trypanosomes}

The antigenic relationships of the trypanosomes were determined mainly by agglutination tests, but serum neutralization tests were used in part of the work.

Agglutination tests. Antigens for agglutination tests were prepared from syringepassaged lines of the isolates by the method of Cunningham \& Vickerman (I962), and stored at $-80^{\circ}$. In a few instances, antigens were also prepared from cyclically transmitted trypanosomes with basic strain antigens (Gray, 1965).

Agglutinating antisera were prepared by infecting rabbits with syringe-passaged or cyclically transmitted trypanosomes. Antisera obtained from animals which had been infected for 3 to 4 weeks and which had high titres of agglutinins to the predominant variant antigens of the isolates (see Gray, 1966) were used in most of the experiments, but pools of sera collected from animals in the second, third and fourth weeks of infection were used occasionally. All antisera were stored at $-20^{\circ}$. 
Agglutination tests were carried out by the method of Cunningham \& Vickerman (I962) with minor modifications (Gray, I962). In the description of the results of the tests the term 'strain' has been used to include all trypanosome isolates and derived clones which produced similar agglutinogenic variant antigens in the first 3 to 4 weeks of infections in rabbits.

Neutralization tests. Antigens for neutralization tests were prepared by diluting antigens for agglutination tests, containing approximately $\mathrm{I}$ to $3 \times 10^{8}$ live organisms $/ \mathrm{ml}$, I/ 100 with a balanced solution of inorganic salts buffered at $\mathrm{pH} 8.0$ by a borate buffer system (Lumsden et al. 1965).

Neutralizing antisera were obtained from rabbits which had been infected with trypanosomes transmitted by tsetse flies. The rabbits were bled for normal serum before infection and for antiserum i4 days afterwards. Neutralizing antisera were also prepared in rats. Groups of twenty 100 to $150 \mathrm{~g}$. rats were infected with approximately $2 \times 10^{6}$ trypanosomes from frozen stocks of appropriate isolates. The rats were treated 3 days later, when the parasitaemias were heavy, with diminazene aceturate $(0.5 \%$, $\mathrm{w} / \mathrm{v}$ ) in distilled water at a dose rate of $2.0 \mathrm{mg}$./ $100 \mathrm{~g}$. rat, administered by intraperitoneal injection. A group of uninfected rats was treated with the drug at the same dose rate to provide control serum. The rats were bled for serum 9 days after drug treatment.

Neutralization tests were performed by the method of Cunningham \& van Hoeve (1963). Normal sera and antisera were diluted I/Io with the borate-buffered balanced salts solution described by Lumsden et al. (I965), chilled to $\circ$ to $2^{\circ}$ and dispensed in $\mathrm{I} \cdot 8 \mathrm{ml}$. volumesin rows of eight tubes. Volumes of $0.2 \mathrm{ml}$. of the suspension of trypanosomes used as the antigen were added to the first tubes in each row and left for $30 \mathrm{~min}$. at $\circ$ to $2^{\circ}$. Seven tenfold serial dilutions of the suspensions of trypanosomes after exposure to the sera and antisera were then prepared and $0 . \mathrm{I} \mathrm{ml}$. volumes of each dilution were inoculated into groups of six mice which were examined for 8 days for trypanosome infections. The infectivity of each test suspension, expressed as the number of $63 \%$ infective doses $\left(\mathrm{ID}_{63}\right)$ per $\mathrm{ml}$., was then estimated from the proportion of mice infected of those inoculated at each dilution by reference to tables published by Lumsden et al. (1963).

\section{RESULTS}

The relationships of trypanosomes isolated from different animals in individual surveys

When two or more isolates of Trypanosoma brucei were obtained from different cattle in a survey they were compared by agglutination tests to find if the animals were infected with related or unrelated organisms.

Survey 5. Isolates $5 / 28$ and 5/65 were virulent when they were first isolated and they adapted to maintenance in rats and mice after 8 and Io passages, respectively. They were transmitted in the laboratory by Glossina morsitans to obtain trypanosomes with basic strain antigens for use in agglutination tests. Tests with these antigens and antisera from rabbits which had been infected with the trypanosomes transmitted by both syringe passage and by tsetse flies for periods up to 4 weeks did not reveal any antigenic relationship between the isolates.

Survey 17 . Six isolates were obtained in survey 17 . Isolates $17 / 14,17 / 30,17 / 70$ and $17 / 96$ adapted readily to maintenance in rats and mice after 8 to Io passages, but isolates $17 / 8$ and $17 / 58$ were much less virulent and caused only light, relapsing parasitaemias after prolonged passage. 
Clones were prepared from the six isolates. Those derived from isolates $17 / 14,17 / 30$, $17 / 70$ and $17 / 96$ were cyclically transmitted to provide trypanosomes with basic strain antigens. Agglutination tests with antisera from rabbits infected by tsetse flies with these four clones, and antisera from rabbits infected by syringe passage with all six clones, showed that clones $17 / 14,17 / 30,17 / 70$ and $17 / 96$ produced variant antigens in common and thus probably originated from the same strain, but clones $17 / 8$ and $17 / 58$ were antigenically distinct. A fuller account of the antigenic relationships of this group of isolates has been given elsewhere (Gray, 1966).

Survey 30 . Twenty-eight isolates were obtained in survey 30 , and 27 were adapted to rats and mice by repeated passage. The number of passages required for the adaptation of different isolates varied from 7 to 28 (average 15 ) and the time required for adaptation varied from 37 to I 53 (average 88 ) days. The exceptional isolate, 30/53, had not adapted completely to rats and mice after 48 passages extending over 203 days. Antisera were prepared by infecting rabbits with syringe-passaged trypanosomes of each isolate, harvested from the blood of infected rats after 3 to 6 passages, when the trypanosomes were still incompletely adapted. Antigens for agglutination tests were prepared after further passage when the isolates were fully adapted to rats and mice.

Antisera obtained from rabbits after 3 weeks of infection were diluted $1 / 40$ and tested for agglutinins to antigens of 27 of the 28 isolates. The antisera could not be tested for agglutinins to isolate $30 / 53$ because the isolate had not adapted to rats. All of the antisera agglutinated three or more of the antigens, indicating that many of the trypanosomes were antigenically related (see Table 2). However, the isolates had probably been derived from only three trypanosome strains each of which produced a number of characteristic variant antigens. These strains have been called Strains I, 2 and 3 for ease of reference in the following account.

Sixteen isolates were collected from cattle infected with trypanosomes of apparently just one of the three different strains. Three isolates were classified as Strain I, two as Strain 2 and I I as Strain 3. Antisera to isolates of Strain I did not agglutinate pure isolates of Strains 2 and 3; antisera to isolates of Strain 2 did not agglutinate pure isolates of Strains I and 3; and antisera to isolates of Strain 3 did not agglutinate pure isolates of Strains I and 2.

Ten isolates were collected from cattle infected with trypanosomes of apparently at least two strains. In nine instances the evidence for the mixed composition of the isolate was clear; either the antiserum to the isolate agglutinated pure isolates of two different strains or the antigen of the isolate was agglutinated by antisera to pure isolates of two strains. Antiserum to isolate 30/94, however, agglutinated isolates of Strain 2 whereas the antigen of the isolate was agglutinated only by antisera to isolates of Strain 3. Also the antisera to three mixed isolates, 30/69, 30/94 and 30/97, failed to agglutinate the homologous trypanosomes. In all, two isolates seemed to consist of organisms of Strains I and 2, three isolates seemed to be a mixture of Strains I and 3, and five a mixture of Strains 2 and 3. There was no strong evidence that any of the isolates were a mixture of trypanosomes of all three strains, but the reaction of the antiserum to isolate $30 / 14$ (probably a mixture of Strains I and 2) with one antigen of Strain 3, and the reaction of the antiserum to isolate $30 / 42$ (probably a mixture of Strains $I$ and 3) with one antigen of Strain 2, indicated that this was a possibility.

The trypanosomes of the remaining antigen $30 / 20$ were lysed in heat-inactivated normal rabbit serum and in antisera to 26 of the isolates, and the isolate could not be 


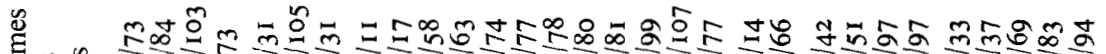

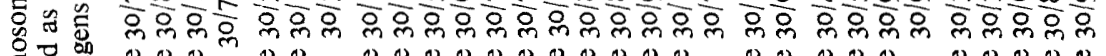

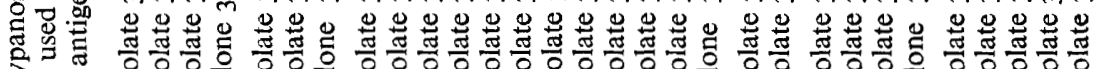


properly classified. However, the antiserum to isolate $30 / 20$ reacted with a few of the antigens of the other isolates and it may have been related to isolates of Strains $I$ and 2. Similarly, reactions of the antiserum to isolate $30 / 53$ with heterologous antigens indicated that isolate 30/53 was related to isolates of Strain 3.

To extend these observations, clones were prepared from isolates 30/73, 30/3I and 30/77, which were representative of Strains I, 2 and 3, respectively, and from isolate 30/97 suspected to be a mixture of trypanosomes of Strains I and 3. When the antisera to the isolates were tested for agglutinins to these trypanosomes, clones $30 / 73,30 / 3 \mathrm{I}$ and $30 / 77$ reacted in almost exactly the same way as the original isolates (Table 2). Further tests with antisera from rabbits infected with the three clones confirmed that they consisted of trypanosomes of different strains (Table 3). Tests with the antisera to the original isolates (Table 2) and antiserum from a rabbit infected with clone 30/97 (Table 3) indicated that clone 30/97 was related only to the isolates of Strain 3 and clone $30 / 77$, unlike the original isolate $30 / 97$ which was agglutinated by antisera to isolates of Strains I and 3.

Table 3. Agglutination of clones prepared from four isolates of Trypanosoma brucei collected in survey 30 by antisera prepared in rabbits (the antisera were tested at $a$ dilution of $1 / 40$ only)

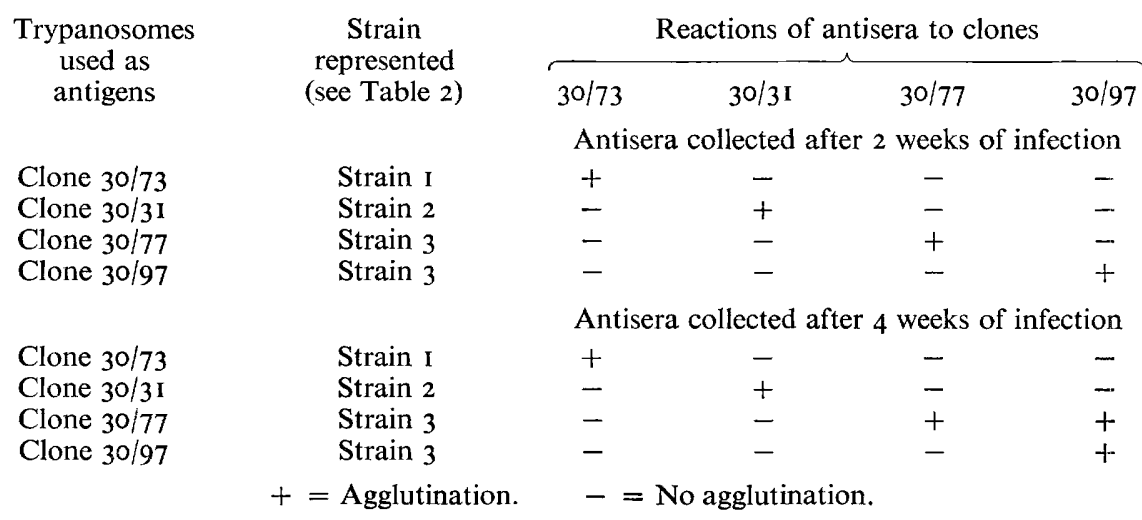

The relationships of trypanosomes isolated in different surveys

At intervals during this work, newly isolated trypanosomes were compared serologically with those collected in earlier surveys.

The relationship between isolates 5/65 and I7/14. A close relationship was found between isolate $5 / 65$, collected from an infected ox in November I96I, and the clone prepared from isolate $17 / 14$, collected from a different animal in November 1963. Agglutinating antisera from rabbits infected with either the isolate or the clone had high titres of antibodies to both antigens (Table 4), which indicated that the trypanosomes were derived from the same strain. The antigenic similarity of these trypanosomes, isolated from the herd at an interval of two years, was of considerable epidemiological significance and the relationship was investigated in greater detail. A clone was prepared from a line of isolate $5 / 65$ which had been maintained first at $-80^{\circ}$ for I 5 months and subsequently in an ox for 9 months. Clones 5/65 and I7/14 were then cyclically transmitted by Glossina morsitans to rats from which trypanosomes with basic strain antigens were obtained and stored at $-80^{\circ}$ as antigens for agglutination 
tests. Rabbits were also infected with the clones by the tsetse flies to provide agglutinating and neutralizing antisera.

Agglutination tests with antisera taken from the rabbits after 10 and 14 days of infection confirmed earlier indications of the close relationship between the isolates and indicated that the cyclically transmitted trypanosomes of the derived clones had antigens in common.

Table 4. Agglutination of isolates of Trypanosoma brucei collected from a herd of cattle over a period of five years by antisera prepared in rabbits

\begin{tabular}{|c|c|c|c|c|c|c|c|c|c|}
\hline \multirow{2}{*}{$\begin{array}{c}\text { Trypanosomes } \\
\text { used as } \\
\text { antigens }\end{array}$} & \multicolumn{9}{|c|}{$\begin{array}{l}\text { Titres of agglutinins in antisera to isolates and clones } \\
\text { (as reciprocals of serum dilutions) }\end{array}$} \\
\hline & $\begin{array}{l}\text { Isolate } \\
5 / 28\end{array}$ & $\begin{array}{l}\text { Isolate } \\
5 i 65\end{array}$ & $\begin{array}{l}\text { Clone } \\
17 / 14\end{array}$ & $\begin{array}{l}\text { Clone } \\
17 / 8\end{array}$ & $\begin{array}{l}\text { Clone } \\
17 / 58\end{array}$ & $\begin{array}{l}\text { Isolate } \\
22 / 24\end{array}$ & $\begin{array}{c}\text { Isolate } \\
30 / 73\end{array}$ & $\begin{array}{c}\text { Isolate } \\
30 / 31\end{array}$ & $\begin{array}{c}\text { Isolate } \\
30 / 77\end{array}$ \\
\hline Isolate $5 / 28$ & 1280 & . & . & . & . & . & . & . & . \\
\hline Isolate $5 / 65$ & . & 1280 & 2560 & . & . & . & . & 20 & . \\
\hline Clone $17 / 14$ & . & 640 & 2560 & . & . & - & . & 20 & . \\
\hline Isolate $22 / 24$ & . & . & . & . & . & 2560 & . & . & . \\
\hline Isolate $30 / 73$ & . & . & . & . & 40 & · & 320 & · & . \\
\hline Isolate $30 / 3 \mathrm{I}$ & . & . & . & . & . & . & . & 2560 & • \\
\hline Isolate $30 / 77$ & . & . & . & . & . & . & . & . & 1280 \\
\hline
\end{tabular}

Table 5. The effect of neutralizing antisera from rats and rabbits on the infectivity of suspensions of trypanosomes of clone 5/65 and clone 17/14

Infectivity of trypanosomes after incubation in normal sera or antisera*

Origin of sera

Normal rabbits

Rabbit infected with clone 5/65

Rabbit infected with clone $17 / 14$

Normal rats

Rats immunized against clone 5/65

Rats immunized against clone $17 / 14$

$\overbrace{\text { Clone } 5 / 65 \text { Clone } 17 / 14}$

$\begin{array}{cc}4 \cdot 8 \pm 0.3 & 5 \cdot 1 \pm 0.5 \\ \text { None } & I \cdot 8 \pm 0.3 \\ 2 \cdot 0 \pm 0.3 & \text { None } \\ 4.9 \pm 0.5 & 5.3 \pm 0.5 \\ 0.8 \pm 0.5 & 1 \cdot 7 \pm 0.3 \\ 2 \cdot 1 \pm 0.3 & \text { None }\end{array}$

* Expressed as the $\log _{10}$ of the number $\mathrm{ID}_{63}$ per $\mathrm{ml}$. of suspension and the standard errors according to Lumsden et al. (1963).

In neutralization tests, antiserum obtained from a rabbit which had been infected with clone $5 / 65$ for I4 days neutralized the infectivity of a suspension of cyclically transmitted trypanosomes of clone $5 / 65$ and considerably reduced the number of infective organisms in a suspension of clone I7/I4. A comparable result was obtained when antiserum from the rabbit infected with clone $17 / 14$ was tested for neutralizing activity against trypanosomes of both clones (Table 5). Further tests with neutralizing antisera prepared by infecting rats with cyclically transmitted trypanosomes of the clones and treating them with therapeutic doses of diminazene aceturate confirmed these findings. Both the rat and the rabbit antisera reduced the infectivity of suspensions of the heterologous clone to approximately the same value which indicated that the cyclically transmitted population of each clone probably consisted of trypanosomes with at least two variant antigens, only one of which occurred in both clones. 
Production of variant antigens by the clones was also compared in chronic infections in rabbits. The rabbits were infected with $2 \times 10^{6}$ cyclically transmitted trypanosomes from the frozen clone stocks and three variant populations of each clone were isolated by injecting $0.5 \mathrm{ml}$. of infected rabbit blood into mice at the ends of the first, second and third weeks of infection. The variants isolated from the rabbit infected with clone $5 / 65$ were designated $5 / 65 \mathrm{a}, 5 / 65 \mathrm{~b}$ and $5 / 65 \mathrm{c}$ and those from the animals infected with clone $17 / 14,17 / 14 \mathrm{x}, 17 / 14 \mathrm{y}$ and $17 / 14 \mathrm{z}$. Sera were collected from the rabbits before infection, at 2-day intervals during the first week of infection and at 7 -day intervals for a further three weeks. The sera were tested for agglutinins to both clones and the six derived variants.

Antibody production by a rabbit infected with clone $5 / 65$
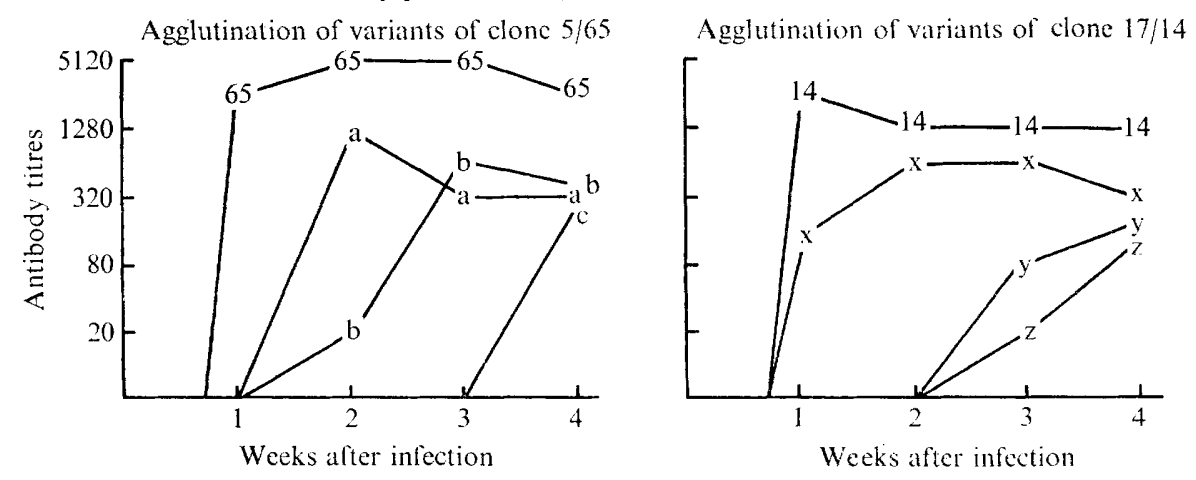

Antibody production by a ribbit infected with clone $17 / 14$

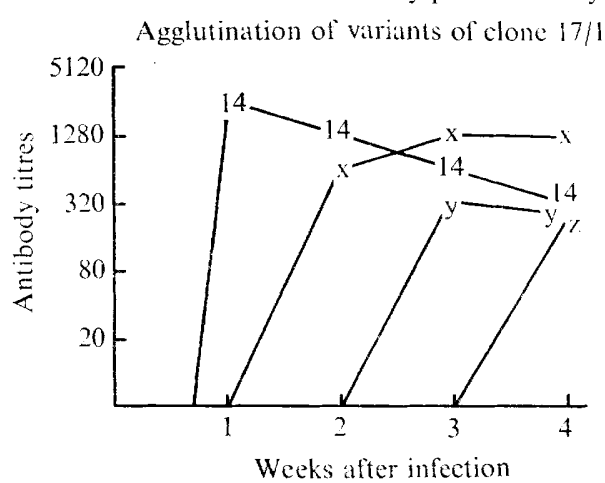

Agglutination of variants of clone $5 / 65$

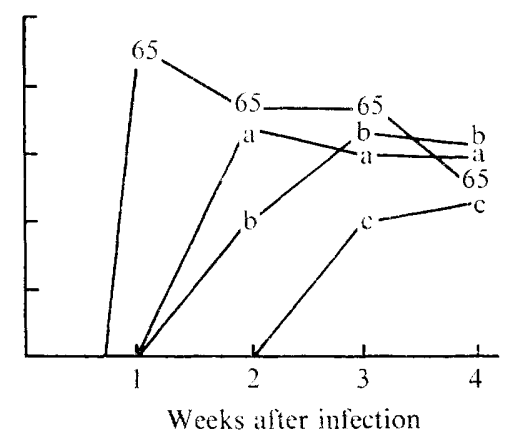

Fig. I. The antigenic similarity of two clones of Trypanosoma brucei illustrated by comparing the production of agglutinating antibodies by rabbits infected with clone $5 / 65$ and clone $17 / 14$ to antigens prepared from the clones and derived variants. $65-65, \mathrm{a}-\mathrm{a}, \mathrm{b}-\mathrm{b}$ and $\mathrm{c}-\mathrm{c}$, production of agglutinins to clone $5 / 65$ and variants $5 / 65 \mathrm{a}, 5 / 65 \mathrm{~b}$ and $5 / 65 \mathrm{c}$. I4-I $4, \mathrm{x}-\mathrm{x}$, $y-y$ and $z-z$, production of agglutinins to clone $17 / 14$ and variants $17 / 14 x, 17 / 14 y$ and $17 / 14 z$.

The clones produced similar variant antigens during the infections because the antisera from the rabbit infected with clone 5/65 agglutinated the trypanosomes isolated from the rabbit infected with clone I7/I4 and vice versa. The patterns of antibody production in the infected rabbits also indicated that, in general, variant antigens were produced in a similar sequence by both clones (Fig. I). 
Other relationships among the isolates. The results of agglutination tests to determine the general relationships of the trypanosomes isolated in four of the five surveys are shown in Table 4. The main characteristics of eight of the nine isolates used in these tests have already been described. The remaining isolate, $22 / 24$, was the only isolate of Trypanosoma brucei obtained from the herd in survey 22. Clone 17/14 was used to represent the antigenically related isolates $17 / 14,17 / 30,17 / 70$ and $17 / 96$ obtained in survey 17 , and isolates $30 / 73,30 / 3 \mathrm{I}$ and $30 / 77$ were used to represent the three strains of trypanosomes obtained in survey 30 . The antisera used were pools of equal volumes of sera obtained from rabbits infected with the isolates at the ends of the second, third and fourth weeks of infection. Each pool was tested for agglutinins to the homologous and heterologous isolates with the exceptions of the antisera to clones $17 / 8$ and $17 / 58$ which were tested only against heterologous trypanosomes, as the homologous antigens were not available.

There was little evidence of antigenic similarity among the isolates with the exception of the close relationship between isolate $5 / 65$ and clone I7/I4, which has been described above. Low titres of antibodies in the antisera to clone $17 / 58$ and isolate $30 / 3$ I suggested that the former was possibly related to isolate $30 / 73$ and the latter to isolate $5 / 65$ and clone $17 / 14$. No evidence to support these possible relationships was obtained when further samples of antisera to clone $17 / 58$ were tested for antibodies to isolate $30 / 73$ and the related isolates $30 / 84$ and 30/103 (see Table 2), or when a new batch of antiserum to clone 17/14 was tested for agglutinins to antigens of isolate $30 / 3 \mathrm{I}$ and the related isolate $30 / 105$.

\section{DISCUSSION}

This study has shown that a majority of isolates of Trypanosoma brucei can be classified by agglutination tests using antisera to the predominant variant antigens of the trypanosomes to yield results of considerable practical value. In earlier work, such tests were used to demonstrate antigenic differences between isolates of T. brucei from widely separated geographical localities and similarities between trypanosomes isolated from different animals kept in one area (Gray, 1966). In the present work, similar methods have been used to show that 26 of 28 isolates of $T$. brucei obtained from a herd of cattle on one day consisted of trypanosomes of only three antigenically distinct strains. The techniques used were also sufficiently sensitive to suggest that ten isolates had been obtained from cattle infected with two different strains of $T$. brucei at the same time, a finding which was confirmed in one instance when a clone was prepared from a suspected mixed isolate.

Four of the isolates studied differed from the majority of the trypanosomes in some respects. Three did not adapt to maintenance in rats and mice, even after prolonged syringe passage, and the fourth was so sensitive to lysis by inactivated serum from both normal and immune rabbits that it could not be used as an antigen in agglutination tests. Furthermore, antisera to the predominant antigens of the four isolates reacted with few of the antigens of the other trypanosomes. Human trypanosomiasis is endemic in the area in which the trypanosomes were isolated (Duggan, 1962) and it is possible that these unusual isolates were the human pathogen Trypanosoma gambiense rather than T. brucei, particularly since the tsetse flies Glossina palpalis and G. tachinoides, which are normally associated with the transmission of $T$. gambiense in Nigeria, were 
probably also the vectors of bovine trypanosomiasis in the experimental area. The problems involved in accurate determination of the species of pleomorphic trypano somes of the subgenus Trypanozoon isolated from animals are well known (see Ormerod, 1967) and it would be difficult to substantiate the above suggestion without testing the infectivity to man of the four unusual isolates. Typical isolates of $T$. brucei and $T$. gambiense usually differ significantly in a number of ways, notably in their pathogenicity for experimental animals, the production of posteronuclear forms, and their sensitivity to tryparsamide and to the lytic effect of human serum, but tests based on such properties are of limited taxonomic value because they may change during the maintenance of isolates in the laboratory and because trypanosomes with intermediate characteristics occur in the field (Lester, 1933).

The advisability of conducting infectivity tests with poorly characterized trypanosomes in human volunteers is questionable, and no attempt was made to test the infectivity to man of the four unusual isolates. It was simply assumed for the purposes of the experiments that all the trypanosomes were Trypanosoma brucei on the grounds that they were isolated from cattle and that $T$. gambiense has not yet been isolated with certainty from any naturally infected vertebrate host in Nigeria other than man. In view of the recent isolation of the closely related human pathogen $T$. rhodesiense from cattle in East Africa (Onyango, van Hoeve \& de Raadt, 1966), this assumption may have been wrong and it is to be hoped that current research on the antigenic relationships of organisms of the subgenus Trypanozoon will lead to unequivocal methods of differentiating between T. brucei and T. gambiense in the future.

One of the main objectives of the present work was to seek evidence of the antigenic stability of strains of Trypanosoma brucei in their natural environment from year to year. Earlier experiments on antigenic variation have indicated that the characteristic basic and predominant variant antigens of each strain of T. bruce $i$ always develop at an early stage of infection when a strain is transferred to a new host by blood passage or by a tsetse fly (Gray, 1965). Strains might therefore be expected to persist in a recognizable antigenic form in the field despite the extensive antigenic variation which occurs in trypanosomes during infections. The antigenic similarity of isolates $5 / 65$ and 17/14 which were obtained from different animals at an interval of 2 years has afforded direct evidence in support of this suggestion. Although the populations of trypanosomes used in the comparative tests were not immunologically identical, the results of the investigation left little doubt that the isolates belonged to the same strain. Despite a careful search, no other examples of such long-term antigenic continuity were detected, but the single positive finding has emphasized the value of serological methods for comparing trypanosomes in epidemiological studies.

The significance of the antigenic diversity of the strains isolated during the 5-year observation period remains to be considered. There were at least seven different strains of trypanosomes among the 37 isolates examined and even more strains might have been found if a more representative sample of the trypanosomes infecting the herd in the first two years of the experiment had been available for study. Only two isolates were available from 20 infected animals in 196I, and none from three animals infected when the herd was examined in 1962 . The policy of treating severely affected animals with therapeutic drugs from time to time also undoubtedly influenced the results by eliminating trypanosomes from the cattle between surveys. The findings that cattle 
kept in a relatively small area under conditions of light tsetse challenge were exposed to infection with at least seven antigenically different strains of one trypanosome species and that animals were occasionally infected with two strains of the same species simultaneously, seem to render the possibility of immunization against trypanosomiasis remote.

I wish to thank Mr C. J. Roberts for much useful discussion and for his continued interest and help during this work.

\section{REFERENCES}

AshCroft, M. T. (I959). A critical review of the epidemiology of human trypanosomiasis in Africa. Tropical Diseases Bulletin 56, 1074-1093.

Broom, J. C. \& Brown, H. C. (1940). Studies in trypanosomiasis. IV. Notes on the serological characters of Trypanosoma brucei after cyclical development in Glossina morsitans. Transactions of the Royal Society for Tropical Medicine and Hygiene 34, 53-64.

CunNingham, M. P. \& van Hoeve, K. (1963). Serological neutralization of trypanosomes. In East African Trypanosomiasis Research Organization Report for 1962-3, p. 24. Edited by J. M. B. Harley. Nairobi: Government Printer.

Cunningham, M. P. \& Vickerman, K. (1962). Antigenic analysis in the Trypanosoma brucei group, using the agglutination reaction. Transactions of the Royal Society for Tropical Medicine and Hygiene 56, 48-59.

DuGGaN, A. J. (1962). A survey of sleeping sickness in Northern Nigeria from the earliest times to the present day. Transactions of the Royal Society for Tropical Medicine and Hygiene 56, 439-486.

GoDFREY, D. G. \& KILLICK-KeNDRICK, R. (I96I). Bovine trypanosomiasis in Nigeria. I. The inoculation of blood into rats as a method of survey in the Donga Valley, Benue Province. Annals of Tropical Medicine and Parasitology 55, 287-297.

Godfrey, D. G., LeACH, T. M. \& KilLICK-KendRick, R. (I964). Bovine trypanosomiasis in Nigeria. III. A high incidence in a group of West African humpless cattle. Annals of Tropical Medicine and Parasitology 58, 204-215.

Gray, A. R.(1962).The influence of antibody on serological variation in Trypanosoma brucei. Annals of Tropical Medicine andParasitology 56, 4-13.

GRAY, A. R. (1965). Antigenic variation in a strain of Trypanosoma brucei transmitted by Glossina morsitans and G. palpalis. Journal of General Microbiology 4I, 195-214.

GraY, A. R. (1966). The antigenic relationship of strains of Trypanosoma brucei isolated in Nigeria. Journal of General Microbiology 44, 263-271.

HOARE, C. A. (1964). Morphological and taxonomic studies on mammalian trypanosomes. X. Revision of the systematics. Journal of Protozoology II, 200-207.

INOKI, S. (1960). Studies on antigenic variation in the Welcome strain of Trypanosoma gambiense. I. Improvements in technique. Biken's Journal 3, 215-222.

Keay, R. W. J. (1953). An Outline of Nigerian Vegetation. Lagos: Government Printer.

LESTER, H. M. O. (1933). The characteristics of some Nigerian strains of the polymorphic trypanosomes. Annals of Tropical Medicine and Parasitology 27, 361-395.

Lumsden, W. H. R. (1965). Biological aspects of trypanosomiasis research. Advances in Parasitology 3, I-57.

Lumsden, W. H. R., Cunningham, M. P., Webber, W. A. F., van Hoeve, K. \& Walker, P. J. (I963). A method for the measurement of the infectivity of trypanosome suspensions. Experimental Parasitology 14, 269-279.

Lumsden, W. H. R., Cunningham, M. P., Webber, W. A. F., van Hoeve, K., Knight, R. H. \& Simmons, V. (1965). Some effects of hydrogen ion concentration on trypanosome numbers and infectivity. Experimental Parasitology 16, 8-17.

OnYANGo, R. J., van HoEve, K. \& DE RAADt, P. (I966). The epidemiology of Trypanosoma rhodesiense sleeping sickness in Alego location, Central Nyanza, Kenya. Evidence that cattle may act as reservoir hosts of trypanosomes infective to man. Transactions of the Royal Society for Tropical Medicine and Hygiene 60, 175-182. 
ORMEROD, W. E. (1967). Taxonomy of the sleeping sickness trypanosomes. Journal of Parasitology $53,824-830$.

TAYLOR, A. E. R. (1968). Immunological studies on the subgenus Trypanozoon and Trypanosoma lewisi. Transactions of the Royal Society for Tropical Medicine and Hygiene 62, 128-129.

WALKER, P. J. (1966). Freeze preservation of parasitic protozoa. Laboratory Practice 15, 423-426.

WeITZ, B. (1962). Immunity in trypanosomiasis. In Drugs, Parasites and Hosts. A Biological Council Symposium, pp. 180-195. London: J. \& A. Churchill. 